\title{
A Fast Tracking Algorithm for the ATLAS Level 2 Trigger
}

\author{
N. Konstantinidis, M. Sutton \\ University College London, Dept of Physics and Astronomy, Gower Street, \\ London, United Kingdom \\ J. Baines, D. Emeliyanov \\ Rutherford Appleton Laboratory, Chilton, Didcot, United Kingdom \\ F. Parodi, C. Schiavi \\ INFN, Genova, Italy \\ H. Drevermann \\ CERN, Geneva, Switzerland
}

\begin{abstract}
A set of conceptually simple and robust algorithms for fast pattern recognition and track reconstruction using 3D space points developed for the ATLAS Level 2 (LVL2) Trigger at the LHC is presented. Results on execution time and physics performance demonstrating the effectiveness of this approach in a demanding, online trigger environment are presented. Both this strategy and a complimentary lookup table based strategy provided results appearing in the recent ATLAS High-Level Trigger, Data Acquisition and Controls Technical Design Report and were successfully implemented online during data taking for the recent ATLAS combined test beam.
\end{abstract}

Key words: tracking, trigger

PACS: 29.40.Gx, 29.40.Wk, 07.05.Kf, 07.05.Hd

\section{Introduction}

The large event rate of the Large Hadron Collider (LHC) makes the online selection of interesting physics events an essential and challenging ingredient required to achieve the physics goals of the LHC. The $40 \mathrm{MHz}$ bunch crossing 
rate of the LHC means that a rejection factor of more than five orders of magnitude is required to reduce the output rate of the ATLAS high level trigger to the $200 \mathrm{~Hz}$ that can be written and stored offline.

For the initial "low" luminosity $\left(2 \times 10^{33} \mathrm{~cm}^{-2} \mathrm{~s}^{-1}\right)$ phase of LHC operation each bunch crossing will produce around five separate minimum bias $p p$ interactions. The later "high" luminosity phase $\left(10^{34} \mathrm{~cm}^{-2} \mathrm{~s}^{-1}\right)$ will produce around $25 \mathrm{pp}$ interactions per bunch crossing. This means that interesting physics events will usually be overlaid by some number of uninteresting, "pileup" interactions from which they must be disentangled. These pileup interactions increase the occupancy of the detector which is a particular problem for the silicon tracking detectors where occupancy is highest. Hits from the overlying pileup interactions increase the probability of incorrectly assigning pileup hits during the online pattern recognition stages and increase the data volume, readout latency and the reconstruction latency. Therefore it is important that the trigger algorithms are both fast and robust to ensure that rare and interesting events are not lost.

\subsection{The ATLAS trigger and DAQ systems}

To achieve this high level of event rejection, the ATLAS trigger has been designed as a three level trigger system [1]. The first level (LVL1) trigger is a hardware pipelined trigger based on custom electronics with reduced granularity information from the calorimeter and the muon system. This is clocked at the bunch crossing rate and delivers a decision after a fixed latency of $2.5 \mu \mathrm{s}$. During LVL1 processing regions of interest (RoI's) within the detector are identified that may contain features of interest and on a LVL1 accept the data are read out into custom Readout Buffers (ROB's) where they are stored for access by the level 2 (LVL2) system.

The LVL2 system consists of a farm of fast commodity CPU's running custom algorithms. During the LVL2 processing the algorithms have access to the full granularity detector data, but only that within the identified LVL1 regions of interest. This approach reduces the data volume and processing required at LVL2. The maximum LVL2 input rate is around $75 \mathrm{kHz}$ and a maximum output rate is around $1 \mathrm{kHz}$.

On a LVL2 accept, the full event is constructed by the Event Builder which passes the data onto the third level Event Filter (EF). This is also a CPU farm, but running seeded versions of the offline reconstruction algorithms with access to the complete detector data and the full alignment and calibration.

The LVL2 trigger is the first stage where information from the tracking detectors is available. At LVL2 it is possible to combine tracking information from the inner detector with calorimeter or muon system data to enable improved 


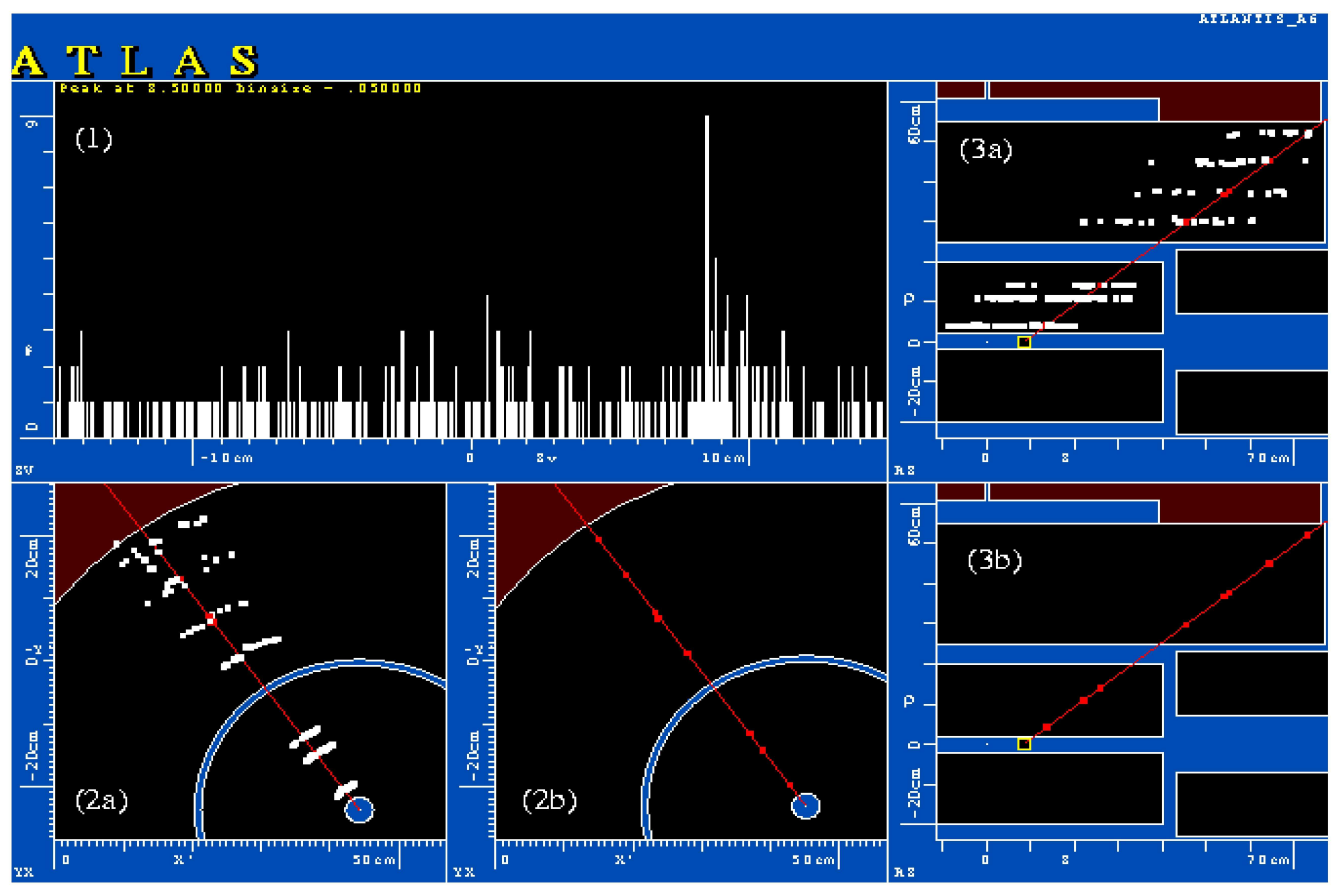

Fig. 1. The ZFinder algorithm for a single electron region of interest.

particle reconstruction, essential for the identification of signatures which may signal new or rare physics, e.g. high transverse energy electrons, photons and muons or final states containing $b$-quarks.

\section{The IdScan level two tracking algorithm}

The IdScan Algorithm [1] attempts to reconstruct with high efficiency high momentum tracks using the the Inner Detector. Throughout, the algorithm uses the ATLAS cylindrical polar coordinate frame with radius $\rho$, polar angle $\phi$ and with $z$ pointing along the beamline. The pseudorapidity $\eta$ is also used for angles with respect to the $z$-axis.

Each $p p$ interaction within a bunch crossing will in general have a different $z$-position along the beamline so that identifying hits consistent with tracks from vertices at different $z$ will reduce the number of hit combinations used for subsequent pattern recognition.

The ZFinder [2] divides the RoI into many small slices in $\phi$. Lower momentum, curved tracks will in general produce small numbers of hits in several $\phi$ slices, whereas those from high momentum straight tracks will produce most of their hits in a small number of neighbouring $\phi$ slices. Within each slice, all possible pairs of space points are then used to calculate the $z$ position of their intersection with the beam line assuming their projection in the $\rho-z$ plane is approximately linear. These $z$ positions are used to fill a one dimen- 
sional histogram for each pair with the histogram maximum corresponding to the $z$ position of the interaction. This is illustrated in Figure 1 for a single high $E_{T}$ electron overlaid with several low momentum pileup interactions. The complete set of space points in the RoI are shown in Figure 1-(2a) and Figure 1-(3a). The electron space points in each of the seven silicon layers of the Inner Detector are shown separately in Figure 1-(2b) and Figure 1-(3b).

This means that for a single electron there are around 21 hit pairs that would contribute to the $z$ vertex in the histogram. This is shown in Figure 1-(1) showing the identified vertex at $z=8.5 \mathrm{~cm}$. For RoI's with more than one track, for example a jet RoI, many more hit pairs pointing to the correct vertex would be expected. By keeping the $\phi$ slices small, most slices have few pairs, and so the algorithm execution time scales approximately linearly with the number of space points.

For single $25 \mathrm{GeV}$ electrons, the $z$-vertex resolution is around $150 \mu \mathrm{m}$ degrading with increasing pseudorapidity. For low luminosity events the efficiency is between 95 and $97 \%$.

Once the $z$ position of the interaction vertex has been identified, the HitFilter then calculates the pseudorapidity, $\eta$, of each space point with respect to this $z$. All the hits in a given RoI are binned in a two-dimensional histogram in $\eta-\phi$ space so that hits consistent with a given high momentum track from that $z$ position would tend to cluster in neighbouring bins of $\eta$ and $\phi$, whereas hits from a track originating at different $z$ would tend to be distributed among many bins at different $\eta$.

The book-keeping monitors the number of different layers that have hits, so that the hits in a cluster of adjacent bins are only considered to belong to a track candidate if hits are observed in at least four out of the possible seven layers. This helps to remove fake candidates. If the bin size in $\eta$ and $\phi$ is small enough, the occupancy of each bin is low. As with the ZFinder algorithm, the execution time scales linearly with the number of space points. The success of the HitFilter depends on the correct $z$ position being used for the pseudorapidity calculation. If the correct position is identified the efficiency of the HitFilter approaches $100 \%$.

After the HitFilter has identified groups of hits which may be consistent with tracks, the GroupCleaner helps to separate hits in clusters which may contain multiple tracks or contain additional hits from unwanted tracks. This is done by taking all possible triplets of hits within the group for a given cluster and using the property that all triplets of hits from the same track should share the same simple track parameters in the transverse plane. The extracted values are then binned in a two-dimensional histogram in $\phi-1 / p_{T}$ space, with combinations containing hits in more than four layers considered as initial sets 
of hits on tracks for input to the Track Fitter.

The Track Fitter [3] uses a Kalman Filter algorithm which estimates the track parameters at the closest approach to the beam line and is able to remove hits from the groups which are not consistent with the track. Further processing is also possible to extend the tracks from the inner silicon layers into the Transition Radiation Tracker for additional pattern recognition and fitting [4].

An alternative approach using lookup tables to identify combinations of hits consistent with high momentum tracks known as SiTrack has also been implemented [1]. This uses space points from any three layers of the silicon detectors. The resulting triplets are fitted as circular arcs in the $\rho-\phi$ plane and straight lines in the $\rho-z$ plane.

\section{Performance}

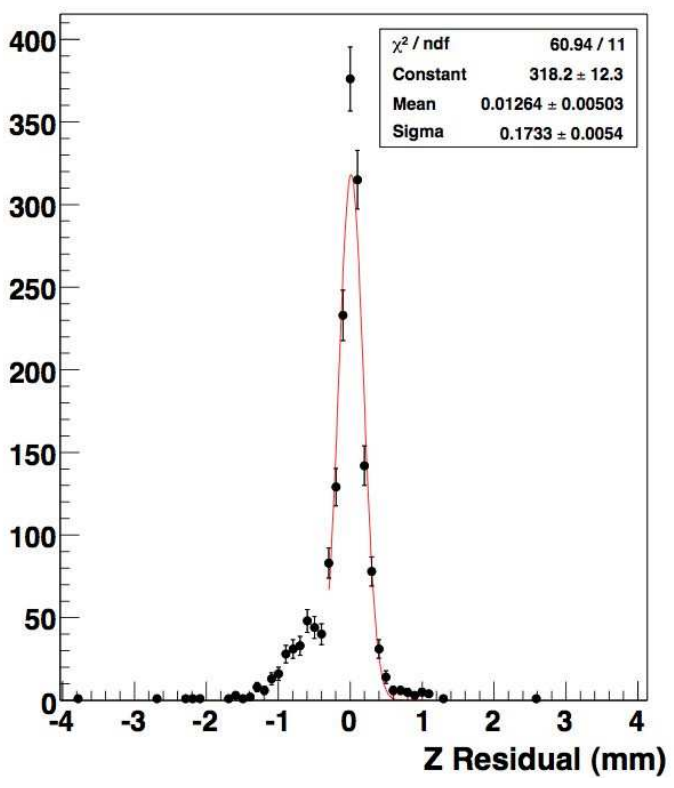

Fig. 2. The IdScan track $z$ residual with respect to the complete offline reconstruction using the combined test beam data.
Studies to evaluate the algorithm performance have been performed. For $40 \mathrm{GeV}$ single electrons with pileup at high luminosity, the mean number of space points within the RoI is around 200 and the mean execution time for the complete IdScan processing on a $1 \mathrm{GHz}$ pentium III is around $1 \mathrm{~ms}$ scaling approximately linearly with the number of space points. The efficiency for electron reconstruction is $95 \%$ and is approximately flat for $|\eta|<2.5$.

For a $B$ physics sample with low luminosity pileup, the mean execution time is around $10 \mathrm{~ms}$ and the vertex resolution for both $b$ - and light quark-jet regions of interest is around $200 \mu \mathrm{m}$. Results from the SiTrack algorithm are very similar to those presented here.

While studies using Monte Carlo simulation are necessary for algorithm evaluation and development, performance studies using real data are also essential. During the end of 2004 a complete section of the ATLAS detector and DAQ system was installed in test beam at CERN. For these studies, the LVL2 tracking algorithms were implemented online and their performance was investigated. For the test beam, IdScan was executed in the normal mode, but 
with a prefilter stage to take account of the large variation $(\sim 1 \mathrm{~cm})$ in the $y$ position of the incident beam, while SiTrack was executed with larger windows.

Figure 2 shows the residual of the reconstructed $z$ position from IdScan with respect to the complete offline Kalman filter track fitter for $40 \mathrm{GeV}$ muons in the solenoidal magnetic field. A central peak with a resolution around $200 \mu \mathrm{m}$ together with a second, smaller peak is observed. These results have been obtained without performing any alignment procedure and so demonstrate that the performance with real data is broadly consistent to that expected from Monte Carlo studies.

The efficiency for reconstructing high energy electrons and muons is above 95\% for both IdScan and SiTrack.

\section{Summary and outlook}

A set of algorithms for the fast reconstruction of high momentum tracks in the demanding high occupancy environment of the silicon tracking detectors in the ATLAS level 2 trigger have been presented.

The performance of the algorithms in terms of reconstruction efficiency, $z$ vertex position resolution and the dependence of the processing time on the data volume is found to be well within the requirements, in the simulation as well as in the ATLAS combined test beam data.

Further development is underway and preliminary studies using IdScan tracks for tagging $B$ mesons using completely reconstructed resonances, secondary vertexing and impact parameter reconstruction appear promising. The algorithms presented have the desired flexibility to cope well with conditions when ATLAS data taking commences in 2007 and to contribute to the success of the ATLAS experiment.

\section{References}

[1] The ATLAS High-Level Trigger, Data Acquisition and Controls Technical Design Report (2003), http://atlas-proj-hltdaqdcs-tdr.web.cern.ch

[2] H. Drevermann and N. Konstantinidis; ATLAS internal communication ATLCOM-DAQ-2002-020 (2002).

[3] D. Emeliyanov; ATLAS internal communication ATL-COM-DAQ-2004-012 (2004).

[4] D. Emeliyanov; these proceedings. 